Análisis y Modificación de Conducta, 1991, Vol. 17. N.9 53-54

\title{
EL IMPACTO DE LA MATERNIDAD EN ALGUNAS VARIABLES DE PERSONALIDAD
}

\section{Pelechano \\ M.P. Matud}

Personalidad, Evaluación y Tratamientos psicológicos

UNIVERSIDAD DE LA LAGUNA (Tenerife)

\section{RESUMEN}

Durante un periodo de 8 meses y en tres momentos diferentes del proceso de ser madre (embarazo, hospitalización post-parto y tres meses tras el parto), 127 mujeres que iban a ser madres y un grupo de control de 100 mujeres, completaron dos cuestionarios (locus de control y motivación y ansiedad de ejecución). El grupo de control completó los cuestionarios en el mismo intervalo temporal. Los resultados muestran que las mujeres que acaban de ser madres presentan más reacciones inhibitorias ante el estrés y más control externo que el grupo de control. También se analizó el papel de diversas variables moduladoras, que muestran que las mujeres que no realizan trabajo renumerado fuera del hogar (las amas de casa) y las mujeres con experiencia de parto puntúan de forma más elevada en ansiedad inhibidora del rendimiento y locus de control externo. Respecto al nivel educativo, a mayor nivel educativo se dan menos diferencias significativas con respecto al grupo de control. La edad también influye, dándose menos diferencias en las edades entre 20 y 29 años. Finalmente, presentamos las implicaciones de estos resultados para los programas de intervención en el área de la maternidad.

Palabras Clave: Personalidad, maternidad, locus de control, motivación y ansiedad. 


\section{SUMMARY}

Along 8 months and in three times (pregnancy, post-partum and puerpery), 127 pregnant-women and 100 non-pregnant women completed two questionnaires (locus of control and performance motivation). The control group completed the questionnaires in the same temporal interval. Results demostrate that women who have just become mothers present more inhibitory reactions when facing stress and more external control than the control group. The role of several moderator variables in the above differences has been analysed: women that only worked at home and that had a previous partum showed the highest scores in inhibitory reactions to stressful situations and external control. In regard to the educational level, the higher the educational level the lesser statistically significant differences. The age variable also plays a role, the meager differences are found in the range between 20 and 29 years. Implications of these results for partum training programs are presented.

Key Words: Personality, motherhood, locus of control, motivation and anxiety.

\section{INTRODUCCION}

La gestación y nacimiento de un hijo implica un gran número de cambios para la mujer, que van desde modificaciones fisiológicas (entre las que destacan la sobrecarga fisiológica y el cambio hormonal) hasta sociales (fundamentalmente las relaciones familiares, de pareja y role), cambios que implican un ajuste tanto físico como social y de cuyos concomitantes psicológicos existe múltiple bibliografía, la cual se caracteriza por la confusión y el desacuerdo (Matud, 1988; Wolkind y Zajicek, 1981). Como un ejemplo paradigmático, está la llamada "depresión post-parto", cuya evidencia empírica es escasa (Pelechano y Matud, 1990).

Respecto al estudio de variables de personalidad, la mayor parte de la bibliografía ha estudiado la relación entre dimensiones de personalidad aisladas y tendencias psicopatológicas, habiendo estudiado, por ejemplo, las dimensiones de neuroticismo durante la gestación (Elliot y cols., 1983; Jarrahi-Zadeh y cols., 1969; Kumar y Robson, 1984; Meares y cols., 1976; Pelechano, 1981; Roldan y Baguena, 1987) motivación (Roldan y Baguena, 1987), extraversion (Elliot y cols., 1983; Kumar y Robson, 1984; Pelechano, 1981; Roldan y Baguena, 1987), ansiedad (Ballinger y cols., 1979; Ballinger, 1982; Bridge y cols., 1985; Cox y cols., 1982; Davidson, 1972; Elliot y cols, 1983; Jarrahi-Zadeh y cols., 1969; Kane y cols., 1968; Kendell y cols., 1981; Little y cols., 1981, 1982; Meares y cols., 1976; Roldan y Báguena, 1987; Zajicek y Wolkind, 1978), hostilidad (Ballinger, 1982; Bridge y 
cols., 1985; Davids y Holden, 1970; Hayworth y cols., 1980; Little y cols., 1981, 1982; locus de control (Hayworth y cols., 1980; Little y cols., 1981, 1982) y variables cognitivo-conductuales como autocontrol (O'Hara y cols., 1984) y estilo atribucional (Cutrona, 1983; Manly y cols., 1982; O'Hara y cols, 1982). Sin embargo, se ha ignorado la dinámica experimental de las dimensiones de personalidad a través del embarazo y la maternidad; además, es difícil encontrar estudios en los que se hayan utilizado grupos de control.

El objetivo del presente estudio es la exploración de (i) la dinámica experimental de las dimensiones de ansiedad, motivación de rendimiento y lugar de control en tres momentos diferentes del proceso de ser madre: durante la gestación, en la hospitalización tras el parto y a los tres meses del nacimiento del hijo; (ii) la influencia de variables socioeconómicas y obstétricas en dicho proceso.

\section{METODO, INSTRUMENTOS Y PARTICIPANTES}

El número total de mujeres que participaron en el estudio es de 227, repartidas en dos grupos. Al primero de ellos lo denominamos "experimental" y está formado por 127 mujeres, que fueron entrevistadas en tres ocasiones distintas: durante la gestación (edad gestacional entre 4 meses y medio y ocho meses), durante la hospitalización postparto (entre 24 y 48 horas tras el parto) y a los tres meses tras el parto. El segundo grupo (al que denominamos "control") está formado por 100 mujeres no gestantes que no habían sido madres en al menos un año antes, las cuales fueron entrevistadas en tres ocasiones respetando los mismos intervalos temporales que para el grupo "experimental". Ambos grupos no se diferenciaban significativamente ni en edad, ni en variables socioculturales (nivel de estudios, profesión de la mujer, profesión del marido, estado civil, domicilio) ni en variables obstétricas (número de embarazos, abortos, partos normales, partos distócicos). Las edades de las mujeres oscilaban entre 16 y 42 años, teniendo menos de 20 años el $8 \%$ de la muestra, y 30 años o más aproximadamente la tercera parte de las mujeres. Respecto al estatus laboral, había un número muy similar de mujeres que realizaban trabajos de tipo manual (limpieza, auxiliar de clínica...), no manual (médicos, secretarias...) y amas de casa.

El pase de pruebas a ambos grupos de mujeres fue en entrevista individual, siguiendo la técnica de entrevista estructurada, durante la cual cumplimentaron el cuestionario de "Motivación y ansiedad de ejecución" y el cuestionario de "Locus de control". También se tomaron una serie de datos socioculturales y obstétricos, tanto por medio de la entrevista como a traves de la historia clínica hospitalaria.

EL cuestionario de Motivación y ansiedad de ejecución está formado por 72 elementos con dos alternativas de respuesta (si/no) y ha sido desarrollado, adaptado y validado para la población española por Pelechano (1975) a partir de 
una serie de trabajos de Brengelmann (1960) y Sedlmayr (1969). Aisla seis factores, cuatro de motivación y dos de ansiedad.

El cuestionario de locus de control es un instrumento construído por Pelechano y Báguena (1983a), del que hemos utilizado una versión que consta de 55 elementos, algunos de los cuales han sido entresacados de escalas ya existentes (Rotter, 1966; Nowicki-Strickland, 1973; Reid-Ware, 1974) y otros construídos para cubrir las áreas en que aquéllos se mostraban insuficientes, especialmente en dimensiones relacionadas con el mundo social. Cada elemento tiene cuatro posibilidades de respuesta (nunca, alguna vez, frecuentemente y siempre) y aisla ocho factores empíricos, cinco de control externo y tres de control interno.

\section{RESULTADOS}

Se han realizado análisis diferenciales bivariados tanto intergrupo (entre las mujeres del grupo experimental y control) como intragrupo (entre las tres ocasiones evaluadas). También se han realizado análisis diferenciales en función de las variables que parecen ser más relevantes: profesión, nivel cultural, edad y experiencia de parto.

En el cuadro número 1 se muestran las diferencias significativas intergrupo. Durante la gestación, no existen diferencias significativas entre los grupos experimental y control en ninguno de los factores. Tras el nacimiento del hijo (hospitalización post-parto y seguimiento), las mujeres declaran más ansiedad inhibidora del rendimiento, mayor percepción de control externo en las relaciones sociales con componentes depresivos y fatalistas y mayor percepción de control interno de responsabilidad decisional y previsión de consecuencias. Existen otra serie de percepciones que parecen estar asociadas de forma específica a una situación concreta, percibiendo mayor control externo de suerte situacional durante la hospitalización postparto y mayor sentimiento de falta de control a los tres meses tras el parto.

Respecto al cambio evolutivo (diferencias intragrupo), los datos se muestran en el cuadro número 2. Las mujeres del grupo experimental muestran mayor tendencia a sobrecargarse de trabajo y menos autoexigencia laboral cuando el niño tiene tres meses, y más motivación positiva durante la hospitalización postparto. Respecto a los factores de locus de control, aumenta progresivamente la percepción de control externo en relaciones sociales con componentes depresivos y fatalistas desde la gestación hasta que el niño tiene tres meses, aumenta asímismo la percepción de control interno de responsabilidad situacional y previsión de consecuencias y disminuye el control interno de autocrítica valorativa en el trabajo y en la interacción social. En la hospitalización postparto aumenta el control 
interno de autoconfianza y control verbal, el cual disminuye de forma significativa tres meses después, y disminuye la percepción de control externo de exculpación y recelosidad, el cual alcanza su valor máximo a los tres meses tras el parto. Estas diferencias parecen estar asociadas de forma específica al hecho de ser madre, ya que no se dan en el grupo de control, grupo en el cual los cambios evolutivos afectan únicamente a dos factores: ansiedad inhibidora y el control interno de responsabilidad decisional y previsión de consecuencias, observándose en ambos una disminución de las puntuaciones a medida que pasa el tiempo (o por la repetición de la cumplimentación de las pruebas).

Respecto al papel de variables moduladoras, en el cuadro número 3 están las diferencias en función de la profesión de la mujer. Las amas de casa son el grupo de mujeres que experimenta más alteraciones asociadas a la maternidad, declarando mayor ansiedad inhibidora del rendimiento y mayor percepción de control externo durante todo el proceso. A los tres meses tras el parto es cuando existen más diferencias en la percepción de control, percibiendo más control externo de exculpación y recelosidad y sentimiento de falta de control, con menor control interno de autoconfianza y control verbal, mientras que la percepción de mayor control interno de responsabilidad decisional y previsión de consecuencias y control externo en las relaciones sociales con componentes depresivos y situacionistas, surgen una vez que ha nacido su hijo (fases de hospitalización y seguimiento).

Respecto a las diferencias entre los grupos experimental y control en los grupos de mujeres que realizan trabajo renumerado fuera del hogar, no se dan diferencias significativas entre las mujeres que realizan trabajo de tipo manual, mientras que las diferencias entre mujeres que realizan trabajo no manual son mínimas, declarando las mujeres gestantes menor percepción de control externo de exculpación y recelosidad y mayor control interno de responsabilidad decisional y previsión de consecuencias las mujeres hopsitalizadas tras el parto.

En cuanto al papel del nivel educativo (cuadro número 4), son las mujeres de nivel más bajo aquellas que parecen mostrar ligeramente más variaciones en conexión con la maternidad, mientras que en las mujeres de mayor nivel educativo (estudios universitarios), las diferencias son mínimas, diferencias que se dan únicamente en las percepciones de lugar de control durante la hospitalización post-parto. La percepción de mayor control interno de responsabilidad decisional y previsión de consecuencias y mayor control externo en relaciones sociales con componentes depresivos y situacionalistas a los tres meses del nacimiento del hijo, se dá en las mujeres de nivel cultural medio y bajo, mientras que el el nivel más elevado de ansiedad y autoexigencia laboral tras el nacimiento del hijo se da únicamente en las mujeres de nivel educativo más bajo. 
CUADRO NUMERO 1.- DIFERENCIAS BIVARIADAS SIGNIFICATIVAS ENTRE LOS GRUPOS EXPERIMENTAL Y CONTROL

\section{GRUPOS}

\section{EXPERIMENTAL CONTROL}

FACTORES

\begin{tabular}{ccc}
$(n=127)$ & $(n=100)$ \\
\hdashline$x \quad$ DT & $x \quad$ DT $t$
\end{tabular}

HOSPITALIZACION POST-PARTO

CUESTIONARIO DE MOTIVACION

$Y$ ANSIEDAD DE EJECUCION:

Ansiedad inhibidora del rendimiento

$\begin{array}{lllll}8.36 & 3.32 & 7.32 & 3.10 & 2.42^{*}\end{array}$

\section{LOCUS DE CONTROL:}

Control externo en relaciones sociales

y con componentes depresivos y

fatalistas

Control externo de suerte situacional

Control interno de responsabilidad decisional y previsión de consecuencias

$\begin{array}{rrrrl}31.98 & 7.99 & 29.19 & 7.14 & 2.74^{* *} \\ 8.59 & 2.54 & 7.87 & 2.44 & 2.16^{*} \\ & & & & \\ 23.79 & 3.61 & 22.43 & 3.61 & 2.81^{* *}\end{array}$

\section{SEGUIMIENTO}

CUESTIONARIO DE MOTIVACION $Y$ ANSIEDAD DE EJECUCION

Tendencia a sobrecarga de trabajo.

Ansiedad inhibidora del rendimiento.

$\begin{array}{lllll}3.53 & 2.60 & 2.88 & 2.24 & 2.00^{*} \\ 8.15 & 3.00 & 6.90 & 3.19 & 3.03^{* *}\end{array}$

\section{LOCUS DE CONTROL:}

Control externo en relaciones sociales con componentes depresivos y fatalistas Control interno de responsabilidad decisional y previsión de consecuencias Control externo de sentimiento de falta de control.

$\begin{array}{rrrrr}32.75 & 7.95 & 29.06 & 7.68 & 3.52^{* * *} \\ 24.20 & 3.23 & 22.41 & 4.11 & 3.58^{* * *} \\ 8.92 & 1.93 & 8.43 & 1.68 & 2.02^{*}\end{array}$

NOTA: $n=$ número de mujeres; DT $=$ Desviación típica; $t=t$ de Student; $\left({ }^{*}\right) p<.05 ;\left({ }^{*}\right)$ $\mathrm{p}<.01 ;\left({ }^{* *}\right) \mathrm{p}<.001$. 


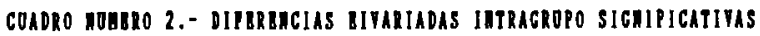

A) GROPO IIPURUBral $(\pi=127)$

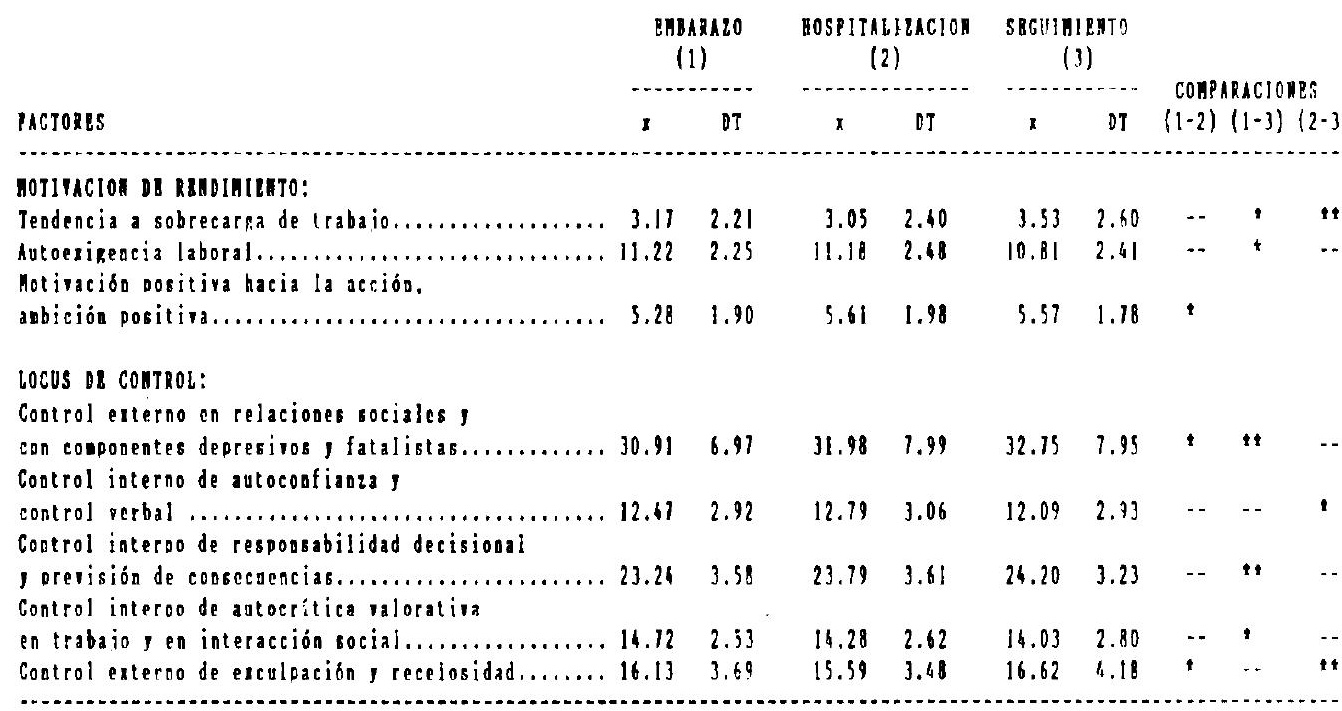

NoTAS: 0 = núbero de sujeres; $x=$ media; $0 T=$ desriación tipica; $t=t$ de studeot; (") $p .(.05:($ ") $)(.01$.

B) Gropo control $(\mathrm{n}=100)$

\begin{tabular}{|c|c|c|c|}
\hline $\begin{array}{c}\text { BABLRAZO } \\
\text { (1) }\end{array}$ & $\begin{array}{l}\text { ROSPITALIRACIOH } \\
\text { (2) }\end{array}$ & $\begin{array}{c}\text { SBCOIMI RSTO } \\
\text { (3) }\end{array}$ & \\
\hline DT & 07 & DT & $(1-2)(1-3)(2-3$ \\
\hline
\end{tabular}

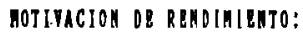

Ansiedad inhibidora del reodinieato.

$\begin{array}{llllll}7.61 & 3.13 & 1.32 & 3.10 & 6.90 & 3.19\end{array}$

LOCOS OE COMTROL:

Control interno de responsabilidad decisional

Iprevisión de consecueveias....................23.75 $3.67 \quad 22.43 \quad 3.61 \quad 22.414 .11$ " 4 " -.

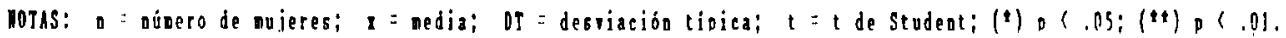


CUADRO NUMERO 3.- DIFERENCLAS BIVARIADAS SIGNIFICATIVAS ENTRE LOS GRUPOS EXPERIMENTAL Y CONTROL MODULADOS SEGUN LA PROFESION DE LA MUJER

A) AMAS DE CASA

EXPERIMENTAL CONTROL

\begin{tabular}{ccc}
$(n=45)$ & $(n=30)$ \\
\hdashline$x$ & DT & $x \quad$ DT
\end{tabular}

FASE DE EMBARAZO:

MOTIVACION DE RENDIMIENTO:

Indiferencia laboral y separación

entre mundo privado y laboral 4.16

1.86

3.03

2.06

$2.45^{*}$

Ansiedad inhibidora del rendimiento .... 9.44

2.83

7.90

3.12

$2.22^{*}$

LOCUS DE CONTROL:

Control externo de suerte situacional .... 9.07

2.96

7.80

2.22

$2.00^{*}$

\section{FASE DE HOSPITALIZACION POST-PARTO \\ MOTIVACION DE RENDIMIENTO:}

Ansiedad inhibidora del rendimiento .... $9.87 \quad 2.73 \quad 7.13 \quad 2.76 \quad 4.22^{* * *}$

LOCUS DE CONTROL:

Control externo en relaciones sociales y

con componentes depresivos y fatalistas 36.11

7.81

28.97

6.57

$4.13^{* * *}$

Control interno de responsabilidad

decisional y previsión de consecuencias 23.62

$3.51 \quad 21.37$

$3.802 .64^{*}$

FASE DE SEGUIMIENTO

MOTIVACION DE RENDIMIENTO:

Ansiedad inhibidora del rendimiento .... 9.53

LOCUS DE CONTROL:

Control externo en relaciones sociales y

con componentes depresivos y fatalistas $\mathbf{3 6 . 6 2}$

7.41

29.47

7.57

$4.06^{* * *}$

Control interno de autoconfianza y

control verbal 10.89

2.92

12.90

$2.31-3.16^{* *}$

Control interno de responsabilidad decisional y previsión de consecuencias. 24.71

2.85

21.73

4.73

$3.10^{* *}$

Control externo de exculpación y

recelosidad 17.87

Control externo de sentimiento de falta de control. 
(CONTINUACION CUADRO NUMERO 3)

B) TRABAJADORAS DE TIPO NO MANUAL

EXPERIMENTAL CONTROL

$$
(n=46) \quad(n=36)
$$

FACTORES

x DT $\quad$ D DT

t

FASE DE EMBARAZO

LOCUS DE CONTROL:

Control externo de exculpación y recelo-

sidad.

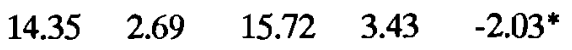

\section{FASE DE HOSPITALIZACION}

\section{POST-PARTO}

LOCUS DE CONTROL:

Control interno de responsabilidad

decisional y previsión de consecuencias. $\begin{array}{llllll}23.83 & 3.57 & 22.17 & 3.19 & 2.19^{*}\end{array}$

NOTAS: $\mathrm{n}=$ número de mujeres; $\mathrm{x}=$ media; $\mathrm{DT}=$ desviación típica; $\mathrm{t}=\mathrm{t}$ de Student. (*) $\mathrm{p}$ $<.05 ;(* *) \mathrm{p}<.01 ;(* *) \mathrm{p}<.001$.

Respecto al papel de la edad de la mujer (cuadro número 5), se da mayor percepción de control externo durante la gestación en las mujeres de edades inferiores a 20 años, dándose mayor percepción de control externo cuando el hijo ha nacido en todos los grupos de edad y mayor control interno de responsabilidad decisional y previsión de consecuencias en las mujeres con edades entre 20 y 29 años. Las mujeres que son madres a mayor edad, declaran más tendencia a la sobrecarga de trabajo en las tres fases, y mayor motivación positiva y más ansiedad facilitadora del rendimiento tras el nacimiento del hijo, mientras que la percepción de mayor control externo tras el nacimiento del hijo se da como un sentimiento de falta de control, mientras que en las mujeres más jóvenes se restringe más a las relaciones sociales, a los que se añade la despreocupación en la planificación de objetivos en las mujeres que son madres antes de los 20 años.

En cuanto al papel de la experiencia de parto (cuadro número 6), parece tener una gran relevancia, dándose una sola diferencia significativa en el grupo de mujeres sin experiencia de parto, mientras que en el grupo de mujeres con experiencia de parto se dan múltiples diferencias significativas. Las mujeres con experiencia declaran mayor ansicdad inhibidora del rendimiento durante las tres ocasiones, mayor tendencia a la sobrecarga de trabajo durante la gestación y el seguimiento, mayor control externo en las relaciones sociales y control interno de responsabilidad decisional y previsión de consecuencias cuando el hijo ha nacido, y mayor sentimiento de falta de control y menor control interno de autoconfianza y control verbal cuando su hijo tiene tres meses. 
CUADRO NUMERO 4.- DIFERENCIAS BIVARIADAS SIGNIFICATIVAS ENTRE LOS GRUPOS EXPERIMENTAL Y CONTROL MODULADOS SEGUN EL NIVEL CULTURAL DE LA MUJER

A) BAJO

EXPERIMENTAL CONTROL

FACTORES

$$
\begin{array}{lll}
(\mathrm{n}=53) & (\mathrm{n}=40) \\
\mathrm{x} & \text { DT } & \mathrm{x} \quad \text { DT }
\end{array}
$$

FASE DE HOSPITALIZACION

\section{POST-PARTO}

MOTIVACION DE RENDIMIENTO

Ansiedad inhibidora del rendimiento. .. 9.41 $3.08 \quad 7.67 \quad 3.14 \quad 2.68^{* *}$

LOCUS DE CONTROL:

Control externo en relaciones sociales

y con componentes depresivos y

fatalistas. 35.898 .56

$32.37 \quad 8.02 \quad 2.01^{*}$

\section{FASE DE SEGUIMIENTO}

MOTIVACION DE RENDIMIENTO:

Autoexigencia laboral. $\begin{array}{lllll}10.47 & 2.17 & 11.60 & 2.16 & -2.49 *\end{array}$

\section{LOCUS DE CONTROL:}

Control externo en relaciones sociales y con componentes depresivos y fatalistas. $\begin{array}{lllll}37.00 & 8.06 & 32.57 & 8.88 & 2.51^{*}\end{array}$

Control interno de responsabilidad decisional y previsión de consecuencias. 24.893 .18

Control externo de exculpación y recelosidad.

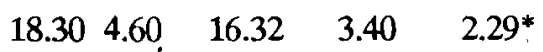


(CONTINUACION CUADRO NUMERO 4)

B)MEDIO

FACTORES

EXPERIMENTAL CONTROL

$$
(\mathrm{n}=42) \quad(\mathrm{n}=28)
$$

$\mathrm{x}$ DT $\mathrm{x}$ DT $\mathrm{t}$

\section{FASE DE EMBARAZO}

LOCUS DE CONTROL:

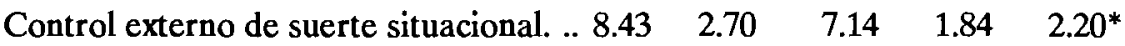

\section{FASE DE HOSPITALIZACION}

\section{POST-PARTO}

LOCUS DE CONTROL:

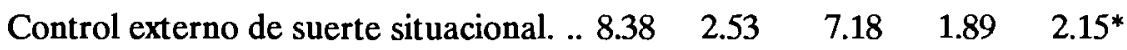

Control interno de responsabilidad

decisional y previsión de consecuencias. $24.76 \quad 3.26 \quad 22.14 \quad 4.58 \quad 2.62^{* *}$

\section{FASE DE SEGUIMIENTO}

LOCUS DE CONTROL:

Control externo en relaciones sociales

y con componentes depresivos y

fatalistas.

$30.95 \quad 5.60$

27.43

6.76

$2.37^{*}$

Control interno de responsabilidad

decisional y previsión de consecuencias. $\begin{array}{lllll}24.29 & 3.27 & 22.11 & 3.14 & 2.77^{* *}\end{array}$

\section{C) ALTO}

EXPERIMENTAL CONTROL

FACTORES

$$
\begin{array}{lll}
(\mathrm{n}=32) & (\mathrm{n}=32) \\
\mathrm{x} & \mathrm{DT} & \mathrm{x}
\end{array}
$$

\section{FASE DE HOSPITALIZACION}

\section{POST-PARTO}

\section{LOCUS DE CONTROL:}

Control interno de responsabilidad

decisional y previsión de consecuencias. $\begin{array}{llllll}23.53 & 3.28 & 21.81 & 2.67 & 2.30^{*}\end{array}$ Control externo de exculpación y recelosidad.

NOTA: $\mathrm{n}=$ número de mujeres; $\mathrm{x}=$ media; $\mathrm{DT}=$ desviación típica; $\mathrm{t}=\mathrm{t}$ de Student. $\left({ }^{*}\right) \mathrm{p}<.05$; (*) $\mathrm{p}<.01$. 
CUADRO NUMERO 5.- DIFERENCIAS BIVARIADAS SIGNIFICATIVAS ENTRE LOS GRUPOS EXPERIMENTAL Y CONTROL MODULADOS SEGUN LA EDAD DE LA MUJER

A) MENOR DE 20 AÑOS

$$
\begin{aligned}
& \text { EXPERIMENTAL CONTROL } \\
& (\mathrm{n}=11) \quad(\mathrm{n}=8)
\end{aligned}
$$

FACTORES

$\begin{array}{llll}\text { x DT } & \mathrm{x} & \text { DT }\end{array}$

$\mathbf{U}$

\section{FASE DE EMBARAZO}

MOTIVACION DE RENDIMIENTO:

Indiferencia laboral y separación entre

mundo privado y laboral 2.09

1.70

2.37

1.92

$15.0^{*}$

LOCUS DE CONTROL:

Control externo en relaciones sociales

y con componentes depresivos y

situacionalistas. $36.09 \quad 5.66$

30.37

3.74

$17.5^{*}$

Control externo de despreocupación

en la planificación de objetivos e insolidaridad pasiva

\section{FASE DE HOSPITALIZACION}

POST-PARTO

\section{LOCUS DE CONTROL:}

Control externo en relaciones sociales

y con componentes depresivos y

situacionalistas.

37.36

7.75

30.37

6.99

$20.0^{*}$

Control interno de autoconfianza y

control verbal.

11.09

2.07

$13.50 \quad 2.14$

$17.0^{*}$

Control externo de despreocupación en la planificación de objetivos e insolidaridad pasiva 
(CONTINUACION CUADRO NUMERO 5)

C) MAYOR DE 29 AÑOS

$$
\begin{array}{cc}
\underset{(n=37)}{\text { EXPERIMENTAL }} & \text { CONTROL } \\
(\mathrm{n}=39)
\end{array}
$$

FACTORES

x DT $\quad \mathbf{x} \quad$ DT

t

FASE DE EMBARAZO

MOTIVACION DE RENDIMIENTO:

Tendencia a sobrecarga de trabajo. ...... $3.59 \quad 1.83 \quad 2.26 \quad 1.79 \quad 3.22^{* *}$

\section{FASE DE HOSPITALIZACION POST-PARTO}

MOTIVACION DE RENDIMIENTO:

Tendencia a sobrecarga de trabajo. ....... 3.54

$\begin{array}{llll}2.54 & 2.00 & 1.79 & 3.04^{* *}\end{array}$

Motivación positiva hacia la acción. ...... 5.46

$\begin{array}{llll}2.12 & 4.51 & 1.96 & 2.03^{*}\end{array}$

Ansiedad facilitadora del rendimiento. 6.89

$\begin{array}{llll}2.83 & 5.38 & 2.82 & 2.33^{*}\end{array}$

\section{LOCUS DE CONTROL:}

Control externo de despreocupación

en la planificación de objetivos e

insolidaridad pasiva 8.38

Control externo de sentimiento de falta

de control.

$\begin{array}{lllll}9.43 & 2.29 & 8.23 & 1.99^{\prime} & 2.44^{*}\end{array}$

\section{FASE DE SEGUIMIENTO}

\section{MOTIVACION DE RENDIMIENTO:}

Tendencia a sobrecarga de trabajo. ...... 3.95

Motivación positiva hacia la acción. ...... 5.43

$\begin{array}{llll}1.83 & 4.51 & 1.80 & 2.20^{*}\end{array}$

Ansiedad facilitadora del rendimiento. 7.03

$3.10 \quad 5.15 \quad 3.03$

$2.66^{*}$

\section{LOCUS DE CONTROL:}

Control externo de sentimiento de falta de control.

$\begin{array}{lllll}9.35 & 2.28 & 8.41 & 1.79 & 2.01^{*}\end{array}$

NOTAS: $\mathbf{n}=$ número de mujeres; $\mathbf{x}=$ media; $\mathbf{D T}=$ desviación típica; $\mathbf{U}=\mathbf{U}$ de Mann-Whitney, $t=t$ de Student. $\left({ }^{*}\right) p<.05 ;(* *) p<.01 ;\left(^{* * *}\right) p<.001$ 
(CONTINUACION CUADRO 5)

\section{FASE DE SEGUIMIENTO}

\section{LOCUS DE CONTROL:}

Control externo en relaciones sociales

y con componentes depresivos y

situacionalistas.

B) ENTRE 20 Y 29 AÑOS

$$
\begin{array}{cc}
\underset{\text { EXPERIMENTAL }}{(\mathrm{n}=79)} & \text { CONTROL } \\
(\mathrm{n}=53)
\end{array}
$$

FACTORES

$\begin{array}{llll}\mathrm{x} & \text { DT } & \mathrm{x} & \text { DT }\end{array}$

\section{FASE DE HOSPITALIZACION}

\section{POST-PARTO}

\section{LOCUS DE CONTROL:}

Control externo en relaciones sociales

y con componentes depresivos y

fatalistas. 30.90

8.09

27.89

6.11

$2.43^{*}$

Control externo de suerte situacional...

$8.23 \quad 2.48$

7.26

2.00

$2.36^{*}$

Control interno de responsabilidad

decisional y previsión de consecuencias. 24.24

3.70

$22.34 \quad 3.66$

\section{FASE DE SEGUIMIENTO}

\section{LOCUS DE CONTROL:}

Control externo en relaciones sociales

$\begin{array}{llllll}\text { y con componentes depresivos y fatalistas.31.54 } & 7.64 & 28.02 & 6.53 & 2.75^{* *}\end{array}$

Control interno de responsabilidad deci-

sional y previsión de consecuencias. ...... $24.25 \quad 3.23 \quad 22.55 \quad 4.40 \quad 2.42^{*}$


El impacto de la matemidad ...

CUADRO NUMERO 6.- DIFERENCIAS BIVARIADAS SIGNIFICATIVAS ENTRE LOS GRUPOS EXPERIMENTAL Y CONTROL MODULADOS SEGUN LA EXPERIENCIA DE PARTO
A) CON EXPERIENCIA

EXPERIMENTAL CONTROL

FACTORES

$$
\begin{array}{lll}
(\mathrm{n}=67) & (\mathrm{n}=76) \\
\mathrm{x} & \text { DT } & \mathrm{x} \quad \text { DT }
\end{array}
$$

t

\section{FASE DE EMBARAZO}

MOTIVACION DE RENDIMIENTO:

$\begin{array}{llllll}\text { Tendencia a sobrecarga de trabajo. ...... 3.37 } & 1.98 & 2.47 & 1.93 & 2.75^{* *} \\ \text { Ansiedad inhibidora del rendimiento. .. 8.82 } & 2.83 & 7.71 & 3.16 & 2.22^{*}\end{array}$

\section{FASE DE HOSPITALIZACION POST-PARTO}

MOTIVACION DE RENDIMIENTO:

Ansiedad inhibidora del rendimiento. .. 8.87 $2.95 \quad 7.09 \quad 3.10 \quad 3.49^{* * *}$

LOCUS DE CONTROL:

Control externo en relaciones sociales

y con componentes depresivos y

fatalistas.

$\begin{array}{llll}33.42 & 7.00 & 29.03 & 7.38\end{array}$

$3.64^{* * *}$

Control interno de responsabilidad

decisional y previsión de consecuencias. 23.72

$\begin{array}{llll}3.78 & 22.29 & 3.81 & 2.24^{*}\end{array}$

\section{FASE DE SEGUIMIENTO}

MOTIVACION DE RENDIMIENTO:

Tendencia a sobrecarga de trabajo. ....... $3.57 \quad 2.30$

$\begin{array}{lll}2.71 & 2.20 & 2.27^{*} \\ 6.74 & 3.27 & 3.04^{* *}\end{array}$

Ansiedad inhibidora del rendimiento. .. 8.28

2.75

LOCUS DE CONTROL:

Control externo en relaciones sociales

y con componentes depresivos y fatalistas.34.19 $6.91 \quad 29.32 \quad 8.05 \quad 3.86^{* * *}$

Control interno de autoconfianza y

control verbal

2.76

12.62

$2.37-2.45^{*}$

Control interno de responsabilidad

decisional y previsión de consecuencias. 24.42

$3.27 \quad 22.09$

$4.28 \quad 3.68^{* * *}$

Control externo de sentimiento de falta

de control.

$\begin{array}{lllll}9.24 & 2.00 & 8.53 & 1.78 & 2.25^{*}\end{array}$


(CONTINUACION CUADRO NUMERO 6)

B) SIN EXPERIENCIA

\begin{tabular}{|c|c|c|c|}
\hline & $\begin{array}{c}\text { EXPERIMENTAL } \\
(n=60)\end{array}$ & $\begin{array}{l}\text { CONTROL } \\
(\mathrm{n}=24)\end{array}$ & \\
\hline FACTORES & x $\quad$ DT & x DT & $\mathbf{U}$ \\
\hline
\end{tabular}

\section{FASE DE HOSPITALIZACION POST-PARTO}

\section{LOCUS DE CONTROL:}

Control externo de exculpación y recelo-

sidad.

NOTAS: $\mathrm{n}=$ número de mujeres; $\mathrm{x}=$ media; $\mathrm{DT}=$ desviación típica; $\mathrm{U}=\mathrm{U}$ de Mann-Whitney; $\mathrm{t}=\mathrm{t}$ de Student. $\left({ }^{*}\right) \mathrm{p}<.05 ;\left(^{* *}\right) \mathrm{p}<.01 ;\left({ }^{* * *}\right) \mathrm{p}<.001$

\section{DISCUSION Y CONCLUSIONES}

Todos estos datos indican que con el nacimiento de un hijo, se dan una serie de modificaciones que parecen centrarse en una mayor percepción de sobrecarga de trabajo y ansiedad inhibidora del rendimiento, así como una mayor percepción de control externo y de control interno de responsabilidad decisional y previsión de consecuencias, percepciones que, en conjunto, sugieren la sobrecarga que parece suponer para la mujer la crianza del hijo.

Este impacto parece estar modulado fuertemente por algunas variables tales como la profesión de la mujer y la experiencia de parto, de forma más moderada, por la edad y por el nivel cultural. El impacto de la maternidad parece ser más elevado en las mujeres de nivel educativo bajo, en las mujeres que no trabajan fuera del hogar y en las mujeres con experiencia de parto, mientras que en las mujeres que realizan trabajo remunerado fuera del hogar, las de nivel cultural alto y las que no tienen experiencia de parto, apenas presentan diferencias significativas respecto al grupo control en las variables estudiadas. La existencia de más alteraciones psicológicas en la vivencia de la maternidad en mujeres con nivel cultural bajo, amas de casa y con experiencia de parto, coincide con los datos encontrados por Pelechano (1981) y Roldan y Báguena (1987). Respecto a la existencia de ansiedad, una de las variables más estudiadas por otros autores, tal y como se vió en la introducción, únicamente se da en estos últimos grupos de mujeres.

La influencia de la maternidad en las variables de personalidad, parece ser 
diferente en función de la edad a la que se es madre, y si bien se observa una tendencia general de aumento en la percepción de control externo tras el nacimiento del hijo, se modifican fundamentalmente las percepciones relacionadas con el lugar de control cuando se es madre a edades muy tempranas, mientras que cuando se es madre a partir de los 30 años, la maternidad supone mayor sobrecarga, pero también más activación y motivación positiva.

La percepción de mayor control externo en las relaciones sociales con componentes depresivos y situacionistas que se da en las tres fases, así como el mayor control externo de despreocupación en la planificación de objetivos e insolidaridad pasiva tanto durante la gestación como inmediatamente tras el nacimiento del hijo, junto a la mayor indiferencia laboral durante la gestación en las mujeres que son madres antes de los 20 años, posiblemente estén reflejando la situación en que se es madre cuando se es aún adolescente: el embarazo y el ser madre es un "accidente" que "ocurrio", sobre el que no se pudo decidir y que coartó en gran medida las posibilidades educativas y profesionales de la mujer. Estos datos deben ser tomados con cierta cautela debido a que únicamente 11 mujeres tenían menos de 20 años, pero sirven al menos para sugerir la problemática asociada con el ser madre muy joven, problemática que parece coincidir con la sugerida por otros autores (Carol y cols., 1980; Petersen y Brooks-Gunn, 1988).

Respecto a las implicaciones para la intervención, estos datos apuntan a la conveniencia de la ampliación de los cursos de psicoprofilaxis obstétrica actuales hasta los tres o cuatro meses tras el nacimiento del hijo, cursos que deberían incorporar estrategias de cambio para estas variables y que deberían tener en cuenta las diferencias citadas, así como la captación de los grupos de mayor riesgo: amas de casa, mujeres con experiencia de parto, mujeres con nivel educativo medio obajo y mujeres muy jóvenes y las que son madres a partir de los 29 años. Respecto a la problemática de la gestación a edades tempranas, la intervención debería incluir la educación sexual y la enseñanza de técnicas anticonceptivas, intervención que debería alcanzar también al periodo de educación obligatoria.

\section{BIBLIOGRAFIA}

BALLINGER, B., BUCKLEY, D. E., NAYLOR, G. H. y STANSFIELD, D. A. (1979).-Emotional disturbance following child-birth: Clinical findings and urinary excretion of cyclic AMP. Psychological Medicine, 9, 293-300.

BALLINGER, C. B. (1982).- Emotional disturbance during pregnancy and following delibery. J. of Psychosomatic Research, 26, 629-634. 
BRIDGE, L. R., LITTLE, B. C., HAYWORTH, J., DEWHURST, J. y PRIEST, R. G. (1985).- Psychometric ante-natal predictors of post-natal depressed mood. J. of Psychosomatic Research, 29, 325-331.

CAROL, C., NADELSON, MALKAH, T., NOTMAN, y GILLON, J. W. (1980).Sexual Knowledge and Attitudes of adolescents: Relationship to Contraceptive Use. Obstetrics and Gyn., 55, 340-345.

COX, J. L., CONNOR, Y. y KENDELL, R. E. (1982).- Prospective study of the psychiatric disorders of childbirth. British J. of Psychiatry, 140, 11-117.

CUTRONA, C. E. (1983).- Causal attributions and perinatal depression. J. of Abnormal Psychology, 92, 161-172.

DAVIDS, A. y HOLDEN, R. H. (1970).- Consistency of maternal attitudes and personality from pregnancy to eigth monts following childbirth. Developmental Psychology, 2, 364-366.

DAVIDSON,J.R.T. (1972).-Post-partum mood change in jamaican women: a description and discussion of its significance. British J. Psychiatry, 121, 659-663.

ELLIOT, S. A., RUGG, A. J., WATSON, J. P. y BROUGH, D. I. (1983).- Mood changes during pregnancy and after the birth of a child. British J. of Clinical Psychology, 22, 295-308.

FLEMING, A. S., RUBBLE, D. N., FLETT, G. L., y SHAUL, D. L. (1988).Postpartum adjustment in first-time mothers: Relations between mood, maternal attitudes, and mother-infant interactions. Developmental Psychology, 24, pp. 71-78.

HAYWORTH, B. C., LITTLE, B. C., CARTER, S. B., RAPTOPOULOS, P., PRIEST, R. G. y SANDLER, M. (1980).- A predictive study of postpartum depression: some predisposing characteristics. British J. of Medical Psychology, 53, 171-167.

JARRAHI-ZADEH, A., KANE, F. J., VAN DE CASTLE, R. L., LACHENBRUCH, P. A. y EWING, A. (1969).- Emotional and cognitive changes in pregnancy and early puerperium. British J. Psychiatry, 115, 797-805.

KANE, F. J., HARMAN, W. J., KEELER, M. H. y EWING, J. A. (1968).Emotional and cognitive disturbance in the early puerperium. British $\mathbf{J}$. Psychiatry, 114, 99-102.

KENDELL, R. E., McGUIRE, R. J., CONNOR, Y. y COX, J. L. (1981).- Mood changes in the first three weeks after childbirth. J. of Affective Disorders, 3, 317-326.

KUMAR, R. y ROBSON, K. M. (1984).- A prospective study of emotional disorders in childbearing women. British J. Psychiatry, 144, 35-47.

LITTLE, B. C., HAYWORTH, J., CARTER, S. B., DEWHURST, J., RAPTOPOULOS, P., SANDLER, M. y PRIEST, R. (1981).- Personal and psychophysiological characteristics associated whit puerperal mental state. J. of Psychosomatic Research, 25, 385-393. 
LITTLE, B. C., HAYWORTH, J., BENSON, P., BRIDGE, L. R.; DEWHURST, J., y PRIEST, R. G. (1982).- Psychophysiological ante-natal predictors of postnatal depressed mood. J. of Psychosomatic Research, 26, 419-428.

MANLY, P. C., McMAHON, R. J., BRADLEY, C. F. y DAVIDSON, P. O. (1982).- Depressive attributional style and depression following childbirth.J. of Abnormal Psychology, 91, 245-254.

MATUD, M. P. (1988).- Depresión post-parto: revisión y datos sobre su existencía y dinámica funcional. Tesis Doctoral. Servicio de publicaciones de la Universidad de La Laguna. Microficha.

MEARES, R., GRIMWADE, J. y WOOD, C. (1976).- A possible relationship between anxiety in pregnancy and puerperal depression. J. of Psychosomatic Research, 20, 605-610.

O'HARA, M. W., CAMPBELL, S. B. y REHM, L. P. (1982).- Predecting depressive symptomatology: Cognitive-behavioral models and postpartum depression. J. of Abnormal Psychology, 91, 457-461.

O'HARA, M. W., NEUNABER, D. J. y ZEKOSKI, E. M. (1984).- Prospective study of postpartum depression: Prevalence, course, and predictive factors. J. of Abnormal Psychology, 93, 158-171.

PELECHANO, V. (1975).- El cuestionario MAE de motivación y ansiedad de ejecución. Fraser S.A.

PELECHANO, V. (1981).- El impacto psicológico de ser madre. Valencia, Alfaplús.

PELECHANO, V. y BAGUENA M. J. (1983).- Un cuestionario de Locus de Control (Lucam). Anal. y modif. de Conducta, 9, 5-46.

PELECHANO, V y MATUD, M. P. (1990).- Depresión post-parto: datos sobre su existencia y codeterminantes en una investigación con grupos criterio y la escala de Beck. Anal. y modif. de conducta, 16, 3-54.

PETERSEN, A. C., y BROOKS-GUNN, J. (1988).- Puberty and Adolescence. En A. P. GOLDSTEIN (ed.).- Handbook of behavioral Medicine for Women. Pergamon Press.

ROLDAN, C. y BAGUENA, M. J. (1987).- Psicología diferencial en la maternidad: datos demográficos y obstétricos como criterios de modulación en dos grupos criterio. Análisis y modificación de conducta, 13, 389-441.

RUBLE, D. N., BROOKS-GUNN, J., FLEMING, A. S., FITMAURICE, G., STANGOR, C., y DEUTSCH, F. (1990).- Transition to motherhood and the self: Measurement, stability and change. J. of Personality and Social Psychology, 58, pp. 450-463.

WOLKIND S. y ZAJICEK, E. (1981).- Pregnancy: A Psychological and Social Study. Academic Press.

ZAJICEK, E. y WOLKIND, S. (1978).- Emotional difficulties in married women during and after the first pregnancy. British J. med. Psychol., 51. 379-385. 